\title{
High Resolution Investigation on the Structure of Quantum Wells in the System CdSe-ZnSe.
}

\author{
H. A. Calderon,* I. Hernandez-Calderon, ${ }^{* *}$ \\ * Escuela Superior de Física y Matemáticas, IPN, UPALM. Ed. \# 9, Zacatenco D.F. Mexico 07738. \\ ** Departamento de Física, CINVESTAV, Mexico D.F. , Mexico.
}

The excitonic emission of ultra-thin quantum wells (1 to 4 monolayers, ML) can be tuned from the yellow-green to the blue spectral range [1]. Therefore they are of interest for the fabrication of light emitting devices. CdSe and $\mathrm{ZnSe}$ have similar crystalline structures (fcc, zinc blende) and differ in the lattice parameter $a$ ( $a$ is 0.567 and $0.608 \mathrm{~nm}$ for $\mathrm{ZnSe}$ and CdSe, respectively). There is therefore a strong lattice mismatch leading to strained interfaces when the QW is sufficiently thin (1 to $3 \mathrm{ML}$ ) and generation of defects above $4 \mathrm{ML}$ of thickness. In this respect one monolayer is the thickness of a cationanion layer and it is given by $a / 2$. Thick QWs (above $4 \mathrm{ML}$ ) have broad and weak emission which is inappropriate for application as light emitting devices. Thus the knowledge of their structure and composition is of prime importance becoming the objective of this investigation with localized microscopy techniques. Previous efforts have concentrated on non-localized characterization techniques such as photoluminescence. Electron microscopes in high resolution modes have been utilized at 300 and $200 \mathrm{kV}$ both in TEM and STEM mode and with the use of aberration correctors. Samples have been prepared by conventional cross section techniques to ensure the highest possible resolution.

Figure 1 shows STEM images of quantum wells in a nominally 15 ML thick QW. The Cd rich QW can be identified both in low magnification (Fig. 1a) and in the high resolution images shown in Figs. 1b,c. The sample orientation [011] allows imaging of dumbbells characteristic of this structure. The $\mathrm{Z}$ contrast is clear enough to determine the QW thickness as $15 \mathrm{ML}$ in average. There is however some distortion and slight overall bending of the QW, most likely due to the sample thinning. The composition is rather homogeneous although with larger variations on the deposit side since $\mathrm{Zn}$ atoms can substitute for $\mathrm{Cd}$ atoms during epitaxial growth. Figure 2 shows STEM images of a QW nominally $3 \mathrm{ML}$ thick. The thickness is around 3ML but the last layer (following the deposit direction) is slightly inhomogeneous. In this thin QWs, it is possible to find deviations from the planarity of the deposits. However they are rather small and most likely mimic the surface of the supporting surface. Additionally there is a small thickness variation that most likely arises from the involved projection along the selected observation direction, too. Direct measurement of chemical composition has been done by using energy loss spectroscopy (EELS). These results support the above conclusions. Transmission electron microscopy with exit wave reconstruction allows imaging of these beam sensitive materials with a slightly better precision and in a shorter time as STEM methods but with a reduction of contrast. Figure 3 shows an example with a direct TEM image. It shows strain contrast (which can be reduced in the EWR procedure) and low contrast. However the QW position and thickness can always be precisely found by measuring the intensity profile (Fig 3b) where a characteristic contrast inversion takes place as a result of Cd substituting for $\mathrm{Zn}$.

References

[1] A. Alfaro-Martínez, I. Hernández-Calderón, Microelectr. J. 36, 362 (2005).

[2] Financial support from IPN (COFAA-SIP), DAAD and CONACYT (grant 51833) are gratefully acknowledged. JEOL, INMETRO (Brazil) and ERZ-Julich FZ are acknowledged 
for the use of Cs probe and Cs image corrected microscopes.
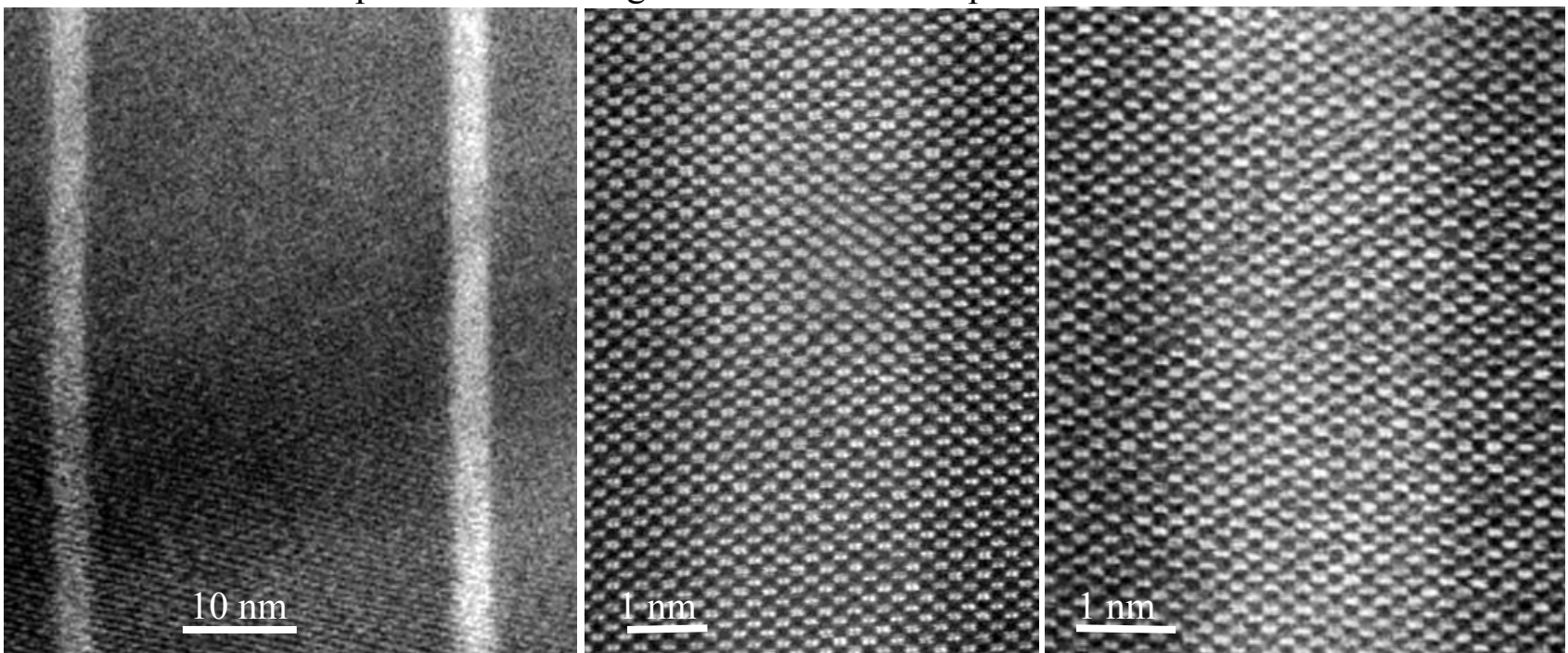

Figure 1. Quantum wells (15 ML) in STEM mode. (a) Low magnification, (b) Thinner and thicker section section of foil. $\mathrm{Z}$ contrast gives location and width of Cd-rich quantum wells.
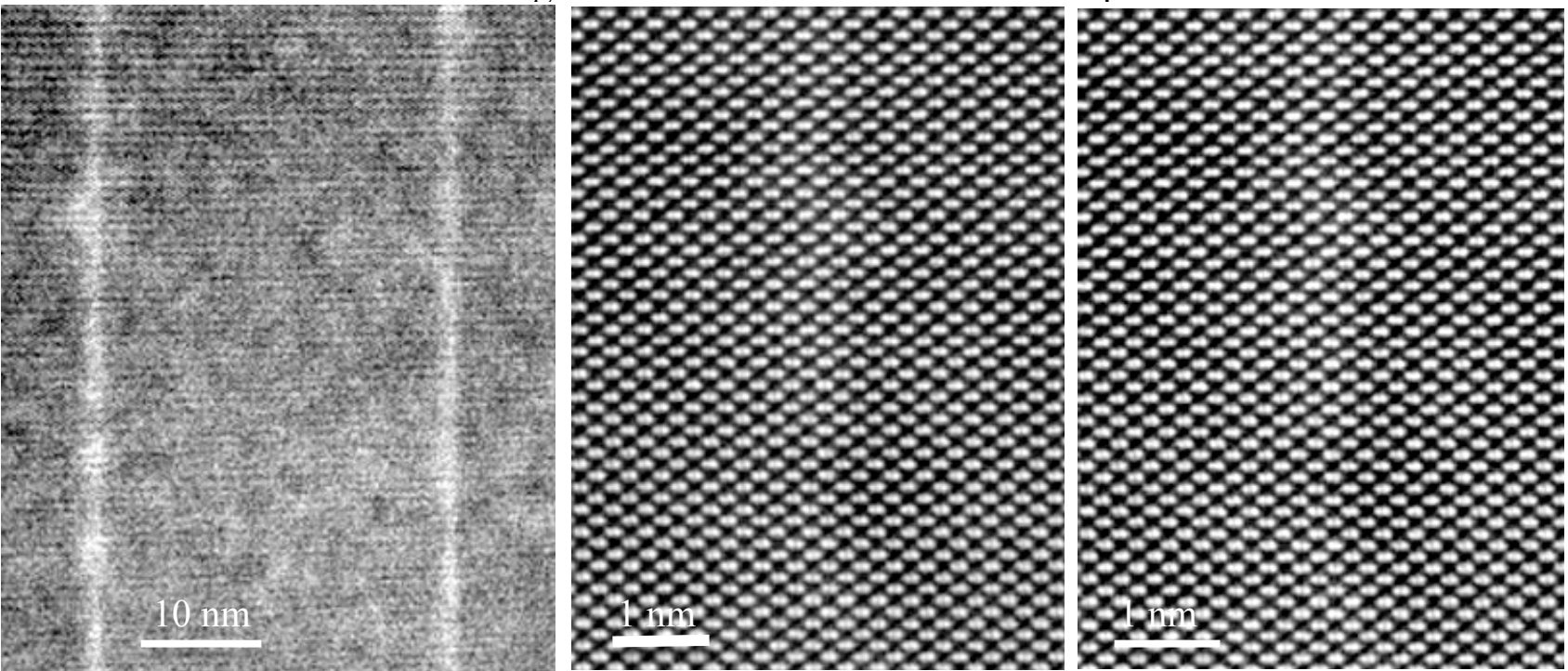

Figure 2. Quantum wells (3 ML) in STEM mode. (a) Low Magnification image, (b) Thinner and slightly thicker (c) section of sample. Z contrast gives location and size of quantum wells.
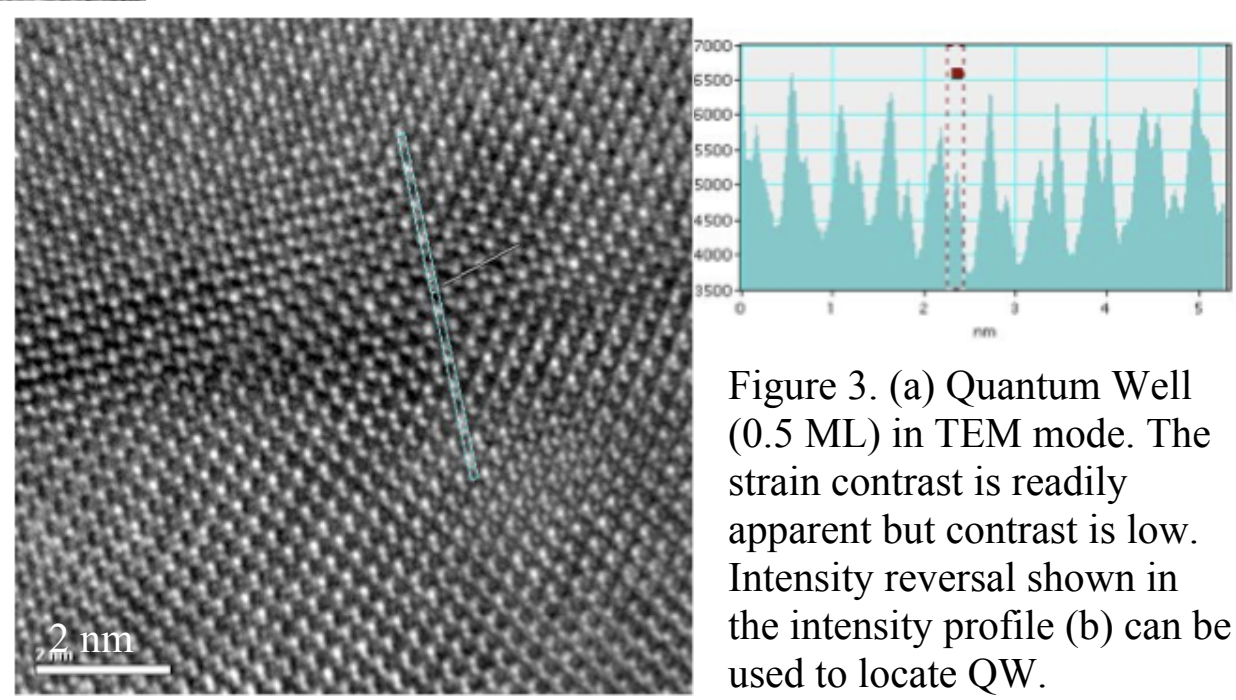

Figure 3. (a) Quantum Well (0.5 ML) in TEM mode. The strain contrast is readily apparent but contrast is low. Intensity reversal shown in the intensity profile (b) can be used to locate QW. 